\title{
Prevalence of vitamin D deficiency and insufficiency among schoolchildren in Greece: the role of sex, degree of urbanisation and seasonality
}

\author{
Yannis Manios $^{1 *}$, George Moschonis ${ }^{1}$, Toine Hulshof ${ }^{2}$, Anne-Sophie Bourhis ${ }^{2}$, George L. J. Hull ${ }^{3}$, \\ Kirsten G. Dowling ${ }^{3}$, Mairead E. Kiely ${ }^{3,4}$ and Kevin D. Cashman ${ }^{3,5}$ \\ ${ }^{1}$ Department of Nutrition and Dietetics, School of Health Science and Education, Harokopio University, 70 El Venizelou \\ Avenue, Kallithea, Athens 17671, Greece \\ ${ }^{2}$ Kellogg Europe, Talbot Road, Stretford, Manchester M16 OPU, UK \\ ${ }^{3}$ School of Food and Nutritional Sciences, Cork Centre for Vitamin D and Nutrition Research, University College Cork, Cork \\ T12 DFK4, Republic of Ireland \\ ${ }^{4}$ Department of Medicine, University College Cork, Cork T12 DFK4, Republic of Ireland \\ ${ }^{5}$ The Irish Centre for Fetal and Neonatal Translational Research (INFANT), University College Cork, Cork T12 DFK4, Republic \\ of Ireland
}

(Submitted 4 May 2017 - Final revision received 28 July 2017 - Accepted 15 August 2017)

\section{Abstract}

The current study was aiming to report the prevalence of suboptimal vitamin D status among schoolchildren in Greece and investigate the role of sex, urbanisation and seasonality on vitamin D status. A sample of 2386 schoolchildren (9-13 years old) from four distinct prefectures was examined. The prevalence of 25-hydroxyvitamin D (25(OH)D) concentration $<30$ and $<50 \mathrm{nmol} / \mathrm{l}$ (vitamin D deficiency and insufficiency respectively) was 5.2 and $52.5 \%$, respectively. Girls had a higher prevalence of $25(\mathrm{OH}) \mathrm{D}<30(7 \cdot 2 v .3 \cdot 2 \%)$ and $50 \mathrm{nmol} / 1(57 \cdot 0 v$. $48 \cdot 0 \%)$ than boys $(P<0 \cdot 001)$. The highest prevalence rates of $25(\mathrm{OH}) \mathrm{D}<30$ and $50 \mathrm{nmol} / \mathrm{l}(9 \cdot 1$ and $73.1 \%$, respectively) were observed during spring (April to June), whereas the lowest ( 1.5 and $31.9 \%$, respectively) during autumn (October to December). The prevalence of $25(\mathrm{OH}) \mathrm{D}<50 \mathrm{nmol} / \mathrm{l}$ was higher in urban/semi-urban than rural regions, particularly during spring months $(74.6 v .47 \cdot 2 \% ; P<0 \cdot 001)$. Female sex, urban/semi-urban region of residence and spring months were found to increase the likelihood of vitamin $\mathrm{D}$ deficiency and insufficiency, with the highest OR observed for spring months (7.47; $95 \%$ CI 3.23, $17 \cdot 3$ and 5.14; $95 \%$ CI 3.84, 6.89 for 25(OH)D <30 and 50 nmol/1 respectively). In conclusion, despite the southerly latitude, the prevalence of low vitamin D status among primary schoolchildren in Greece is comparable to or exceeds the prevalence reported among children and adolescents on a European level. Sub-populations at highest risk are girls in urban/semi-urban areas during spring months, thus indicating the need for effective initiatives to support adequate vitamin D status in these population groups.

\section{Key words: Vitamin D: 25-Hydroxyvitamin D: Sex: Urbanisation degree: Seasonality: Children}

Over several recent decades, vitamin D has been identified as one of the key nutrients that contributes to the development and maintenance of optimum bone mass. Vitamin D primarily enhances intestinal $\mathrm{Ca}$ and phosphate absorption and promotes bone mineralisation, thus supporting optimal skeletal growth and development from the very early life stages ${ }^{(1,2)}$. Over the last 15 years, the attention on vitamin $\mathrm{D}$ and health has increased considerably ${ }^{(3,4)}$, mainly due to the discovery of vitamin $\mathrm{D}$ receptors in most body tissues and cells, which generated new hypotheses on the potential impact of vitamin D on several health outcomes beyond its known metabolic actions on bone and mineral metabolism ${ }^{(5)}$.

Wide variations in the prevalence of poor vitamin D status have been reported across countries throughout the world, which appear to be dependent on latitude, seasonality, the characteristics of the populations under study, the vitamin D food fortification policy in each country, as well as the thresholds of serum 25-hydroxyvitamin D (25(OH)D) concentrations used to define deficiency or insufficiency ${ }^{(6-8)}$. Although the serum thresholds as proposed by the Institute of Medicine (IOM) (i.e. 25 $(\mathrm{OH}) \mathrm{D}<30$ and $50 \mathrm{nmol} / \mathrm{l}$ ) have been most commonly used to represent deficiency and insufficiency, respectively ${ }^{(9)}$, a higher threshold of $75 \mathrm{nmol} / \mathrm{l}$ has also been proposed by the Endocrine Society ${ }^{(10)}$. Based on these thresholds, the prevalence of low vitamin D status among European populations is characterised by a high degree of variability across studies and countries ${ }^{(6,11)}$. In this context, the 'Healthy Lifestyle in Europe by Nutrition in Adolescence' study reported a range in the prevalence of vitamin D

Abbreviations: 25(OH)D, 25-hydroxyvitamin D; HGS, Healthy Growth Study; LC, liquid chromatography; VDSP, Vitamin D Standardization Program.

* Corresponding author: Y. Manios, fax +30 210 9549141, email manios@hua.gr 
insufficiency from 25.7 to $62.9 \%$ among adolescents from nine European countries ${ }^{(12)}$.

Despite the fact that the major natural source of vitamin D in humans is its endogenous synthesis via exposure of the skin to solar UVB radiation, according to country-specific regional data there is a higher prevalence of low vitamin D status recorded among children and adolescents from southern Europe compared with their counterparts from central and northern Europe. For example, recent data suggested that children and adolescents living in Italy and Turkey ${ }^{(13,14)}$, had a similar or higher prevalence of vitamin D insufficiency (at 57.4 and $93 \%$, respectively) compared with children and adolescents from Northern Ireland, the Netherlands and Great Britain (46, 51.3 and 53\%, respectively) during winter and/or spring months ${ }^{(15-17)}$. These findings warrant further investigation, especially for populations with very limited data. Further to variations within the wider European region, the prevalence of low vitamin D status may also vary considerably even within the same country and season, as highlighted by the findings of two different studies conducted among Turkish children and adolescents that reported a prevalence of serum $25(\mathrm{OH}) \mathrm{D}$ concentrations $<50 \mathrm{nmol} / 1$ of 8 and $98.7 \%$, respectively, during winter $^{(18,19)}$. However, it should be noted that serum $25(\mathrm{OH}) \mathrm{D}$ levels in these two studies were measured with two different assays (i.e. chemiluminescence immunoassay and HPLC respectively). Considering the well-recognised method-related differences in the measurement values for circulating $25(\mathrm{OH}) \mathrm{D}^{(20,21)}$, this wide variation could be at least partly due to a lack of a standardisation between the methods applied to determine serum $25(\mathrm{OH})$ D levels in these two studies.

In light of this, the aim of the current study was to report the prevalence of vitamin D deficiency and insufficiency in a representative sample of 2386 children living in four prefectures covering the northern, southern, eastern and central parts of Greece and to further examine the role of sex, degree of urbanisation and seasonality on the vitamin D status of these children. As the first 806 children in the Healthy Growth Study (HGS) were analysed for serum 25(OH)D in 2013 and the remaining 1580 samples were analysed in 2016, it was important to standardise the serum $25(\mathrm{OH}) \mathrm{D}$ data as per the Vitamin D Standardization Program (VDSP) protocols ${ }^{(22)}$ before combining the two data sets.

\section{Methods \\ Study design and population}

The HGS was a large-scale cross-sectional epidemiological study initiated in May 2007 and completed in June 2009. Approval to conduct the study was granted by the Greek Ministry of National Education and the Ethical Committee of Harokopio University of Athens (Reference code: 16/19-122006). The population under study comprised of schoolchildren aged 9-13 years attending the fifth and sixth grades from primary schools located in municipalities within the counties of Attica, Aitoloakarnania, Thessaloniki and Iraklio. The study population was representative of the 9-13-year-old schoolchildren living in the four counties under study, which are scattered throughout the Greek territory, covering the northern (i.e. Thessaloniki), central (i.e. Attica), western (i.e. Aitoloakarnania) and southern (i.e. Iraklio-Crete) parts of Greece (indicating potential representativeness also at a national level). The sampling of schools participating in the HGS was random, multistage and stratified by parents' educational level and total population of students attending schools within municipalities of these counties ${ }^{(23)}$. A detailed letter, explaining the aims of the current study and a consent form for conducting full measurements, was provided to all parents or guardians having a child in these schools. Parents, who responded positively, had to sign the consent form and provide their contact details. Signed parental consent forms for full measurements were collected for 2655 out of 4145 children (response rate $64 \cdot 1 \%$ ).

\section{Measurements}

Socio-economic and demographic indices. Data on the socioeconomic and demographic background of the families having at least one child participating in the study were collected from the parents (most preferably from the mother) during scheduled face-to-face interviews at the school-site. For those parents, who were not able to attend the meetings (approximately $5 \%$ of the total sample), data were collected via telephone interviews. All interviews were conducted by researchers that were rigorously trained to minimise interviewer's effect, with the use of a standardised questionnaire. More specifically, the data collected during the interviews included parental educational level (years of education), origin and nationality.

Dietary intake. Dietary intake data were obtained by trained dieticians and nutritionists, via morning interviews with children at the school sites. Dietary intake data were obtained for 2 consecutive weekdays and 1 weekend day, using the 24-h recall technique. To ensure quality, food models and portion measures (such as cups and spoons) were used to specify serving sizes, a trained dietitian checked the food records for any mis-recorded or missing information, whereas a random $10 \%$ of all records was re-entered in the food database by a different person than the one entering the original data. The data were then compared with the original to ensure interresearcher reliability. Food intake data were analysed with Nutritionist V diet analysis software (version 2.1, 1999; First Databank), which was extensively amended and updated to include traditional Greek foods and recipes, as described in Food Composition Tables and Composition of Greek Cooked Food and Dishes ${ }^{(24,25)}$. Furthermore, the databank was updated with nutrition information of chemically analysed commercial food items, most usually consumed by children in Greece.

Physical activity levels. To assess physical activity, study participants were provided with and instructed to wear a waistmounted pedometer (Yamax SW-200 Digiwalker; Yamax Corporation) for 1 week (i.e. from Monday to Sunday). The pedometer was positioned in accordance with the manufacturer's instructions on the right waistband, vertically aligned 
with the patella. The pedometer used in the present study displayed the cumulative number of steps from the time it was worn in the morning until the time it was removed, just before bedtime. Participants were provided with a diary template and instructed to record the total number of daily steps displayed by the pedometer at bedtime and then zero the pedometer. Participants were also instructed to take off the pedometers when bathing or swimming and to record these activities in the diary.

Physical examination. All children underwent a physical examination by an experienced female paediatrician that assessed pubertal maturation (i.e. by using the Tanner stage) after a visual inspection of breast development in girls and genital development in boys ${ }^{(26)}$.

Anthropometric indices. Anthropometric measurements were conducted by two trained members of the research team. The protocol and equipment used were the same in all schools. Body weight was measured to the nearest $10 \mathrm{~g}$ using a Seca Alpha (model 770) digital scale (Seca North America, Medical Measuring Systems and Scales). Children were weighed without shoes in the minimum clothing possible. Height was measured to the nearest $0 \cdot 1 \mathrm{~cm}$ using a commercial stadiometer (Leicester Height Measure; Invicta Plastics), with the pupil standing barefoot, keeping shoulders in a relaxed position, arms hanging freely and head aligned to Frankfort horizontal plane. Body weight and height measurements were subsequently used to calculate BMI according to Quetelet's equation (body weight $(\mathrm{kg}) /$ height $\left.(\mathrm{m})^{2}\right)$.

Biochemical indices. Blood samples were obtained from all children during morning visits at schools, between 08.30 and 10.30 hours and following a 12-h overnight fast. Reminders were distributed the previous day to both parents and children, in order to ensure compliance with fasting. Professional staff performed venepuncture for all children. Blood was collected in plain test tubes for the preparation of serum, which was divided into aliquots and stored at $-80^{\circ} \mathrm{C}$. Serum $25(\mathrm{OH}) \mathrm{D}$ and intact parathyroid hormone (PTH) levels were measured in all samples using chemiluminescence assay (Elecsys Roche Diagnostics) in a Cobas e411 Elecsys 2010 analyser (HitachiTechnologies Corp.). The serum 25(OH)D analysis was conducted in two batches within the HGS (806 samples in 2013 (referred to as HGS I) and 1580 in 2016 (referred to as HGS II)).

Standardisation of 25-hydroxyvitamin $D$ levels. The $\mathrm{VDSP}^{(22)}$ protocol for standardisation of serum 25(OH)D values from past surveys was applied to serum 25(OH)D data arising from the two separate sets of chemiluminescence immunoassay analysis (i.e. HGS I and II). The VDSP protocol and its application has been outlined in detail elsewhere ${ }^{(22,27-29)}$, but in brief, for each set of serum $25(\mathrm{OH}) \mathrm{D}$ data separately, a subset of bio-banked serum samples were selected by a specific VDSP statistical algorithm, retrieved from the freezers and sent to University College Cork for re-analysis of 25(OH)D levels by their Centre for Disease Control and Prevention-certified liquid chromatography-tandem MS (LC-MS/MS) method, which is traceable to the Reference Measurement Procedures.
The results were used to develop a master regression equation for each wave, which allowed recalibration of the existing 25 $(\mathrm{OH}) \mathrm{D}$ data set for the entire survey sample. The 806 serum samples were standardised as part of the 'Food based solutions for Optimal vitamin D Nutrition and health through the life cycle' (ODIN) project ${ }^{(29)}$, whereas the remaining 1580 serum samples were standardised as part of the present work and once both data sets (HGS I and II) were standardised they were combined. The two master regression equations for the HGS serum $25(\mathrm{OH}) \mathrm{D}$ data are shown in the online Supplementary Table S1.

Categorisation of vitamin $D$ status. Standardised serum $25(\mathrm{OH}) \mathrm{D}$ concentrations were used to categorise children according to their vitamin D status, using the cut-off values proposed by the US IOM Dietary Reference Intake committee's recent definitions: persons are at risk of deficiency at serum $25(\mathrm{OH}) \mathrm{D}$ concentrations $<30 \mathrm{nmol} / \mathrm{l}$, whereas serum $25(\mathrm{OH}) \mathrm{D}$ concentrations $>30$ but $<50 \mathrm{nmol} / 1$ is indicative of insufficiency, as $50 \mathrm{nmol} / 1$ is consistent with that needed by the $97.5 \%$ of individuals aged $>1$ year to support bone health ${ }^{(9)}$. To promote transparent reporting and international comparisons, we have also reported the percentage of children with $25(\mathrm{OH}) \mathrm{D}$ concentration between 50 and $75 \mathrm{nmol} / \mathrm{l}$, as designated by the Endocrine Society to denote insufficiency ${ }^{(10)}$.

\section{Statistical analysis}

Categorical variables are expressed as percentages, whereas continuous variables as means and standard deviations. The $\chi^{2}$ test was used to explore the association between categorical variables, using the two-sample $z$ test for proportions for post hoc multiple comparisons. The Kolmogorov-Smirnov test was used to determine normality of distribution of the examined continuous variables. All continuous variables examined in the present study were found to be normally distributed and as such the Student's $t$ test was used for the comparison of mean values between groups. Multivariate logistic regression analysis was also performed in order to assess the associations of sex, surbanisation degree (i.e. urban, semi-urban or rural) and seasonality with vitamin D deficiency and insufficiency respectively. OR and 95\% CI were also derived from these analyses after adjusting for several potential confounding factors. All statistical analyses were performed with the SPSS statistical analysis software version 23.0. All $P$ values reported were two-tailed and the level of statistical significance was set at $P<0.05$.

\section{Results}

Table 1 summarises the descriptive characteristics of the study population, in the total sample and by sex ( $49.8 \%$ females). The mean age of study participants was 11.2 (sD 0.7) years. Their mean dietary vitamin D intake, physical activity levels and PTH concentrations were $1.55 \mu \mathrm{g} / \mathrm{d}, 13276 \mathrm{steps} / \mathrm{d}$ and $35.2 \mathrm{pg} / \mathrm{ml}$, respectively. In addition, $36.5 \%$ of participants were measured in autumn and $68.1 \%$ resided in large urban/metropolitan areas, particularly Attica/Athens (57.2\%), with both their parents having $<14$ years of education $(52 \cdot 2 \%)$. Almost all $(98 \cdot 1 \%)$ 
Table 1. Descriptive characteristics of study participants

(Mean values and standard deviations; percentages and numbers)

\begin{tabular}{|c|c|c|c|c|c|c|c|}
\hline & \multicolumn{2}{|c|}{ Total sample ( $n$ 2353) } & \multicolumn{2}{|c|}{ Boys ( $n$ 1179) } & \multicolumn{2}{|c|}{ Girls ( $n$ 1174) } & \multirow[b]{2}{*}{$P^{*}$} \\
\hline & Mean & SD & Mean & SD & Mean & SD & \\
\hline Age (years) & $11 \cdot 2$ & 0.7 & $11 \cdot 2$ & 0.7 & $11 \cdot 2$ & 0.7 & 0.750 \\
\hline Dietary vitamin $D$ intake $(\mu \mathrm{g} / \mathrm{d})$ & 1.55 & 1.28 & 1.64 & 1.38 & 1.46 & $1 \cdot 16$ & 0.001 \\
\hline Physical activity level (steps/d) & 13276 & 5262 & 14767 & 5691 & 11773 & 4299 & $<0.001$ \\
\hline \multirow[t]{2}{*}{ Serum PTH $(\mathrm{pg} / \mathrm{ml})$} & 35.2 & $12 \cdot 2$ & 33.0 & $10 \cdot 8$ & 37.5 & $13 \cdot 0$ & $<0.001$ \\
\hline & $\%$ & $n$ & $\%$ & $n$ & $\%$ & $n$ & \\
\hline \multicolumn{7}{|l|}{ Seasonality } & 0.601 \\
\hline Autumn (October to December) & $36 \cdot 5$ & 859 & $36 \cdot 7$ & 433 & $36 \cdot 3$ & 426 & \\
\hline Winter (January to March) & 34.9 & 821 & $34 \cdot 0$ & 401 & $35 \cdot 8$ & 420 & \\
\hline Spring (April to June) & 28.6 & 673 & $29 \cdot 3$ & 345 & $27 \cdot 8$ & 328 & \\
\hline \multicolumn{7}{|l|}{ Region of residence } & 0.193 \\
\hline Central Greece (Attica) & $57 \cdot 2$ & 1346 & $59 \cdot 3$ & 698 & $55 \cdot 2$ & 648 & \\
\hline Southern Greece (Iraklio-Crete) & $16 \cdot 9$ & 398 & $16 \cdot 5$ & 195 & $17 \cdot 3$ & 203 & \\
\hline Western Greece (Aitoloakarnania) & 13.5 & 318 & 13.0 & 153 & $14 \cdot 1$ & 165 & \\
\hline Northern Greece (Thessaloniki) & $12 \cdot 4$ & 291 & 11.3 & 133 & 13.5 & 158 & \\
\hline \multicolumn{7}{|l|}{ Urbanisation degree } & 0.194 \\
\hline Large urban regions & $68 \cdot 1$ & 1602 & $69 \cdot 7$ & 822 & $66 \cdot 5$ & 780 & \\
\hline Semi-urban regions & $15 \cdot 3$ & 360 & $14 \cdot 1$ & 166 & $16 \cdot 4$ & 194 & \\
\hline Rural regions & $16 \cdot 6$ & 391 & $16 \cdot 2$ & 191 & $17 \cdot 0$ & 200 & \\
\hline \multicolumn{7}{|l|}{ Parental education level } & 0.725 \\
\hline Both parents $<14$ years & $52 \cdot 2$ & 1228 & $51 \cdot 8$ & 611 & $52 \cdot 7$ & 617 & \\
\hline One parent $<14$ years & 23.7 & 558 & 23.4 & 276 & 23.9 & 282 & \\
\hline Both parents $>14$ years & $24 \cdot 1$ & 567 & $24 \cdot 8$ & 292 & $23 \cdot 4$ & 275 & \\
\hline \multicolumn{7}{|l|}{ Race } & 0.662 \\
\hline Caucasian & $98 \cdot 1$ & 2308 & 98.0 & 1155 & $98 \cdot 2$ & 1153 & \\
\hline Non-Caucasian & 1.9 & 45 & $2 \cdot 0$ & 24 & 1.8 & 21 & \\
\hline \multicolumn{7}{|l|}{ Origin } & 0.119 \\
\hline Greek & 83.9 & 1974 & $85 \cdot 1$ & 1003 & $82 \cdot 7$ & 971 & \\
\hline Non-Greek & $16 \cdot 1$ & 379 & 14.9 & 176 & $17 \cdot 3$ & 203 & \\
\hline \multicolumn{7}{|l|}{ Body weight status } & 0.001 \\
\hline Under-/normal-weight & 57.8 & 1360 & $55 \cdot 1$ & 650 & $60 \cdot 6$ & 710 & \\
\hline Overweight & 30.5 & 718 & 31.0 & 365 & $30 \cdot 1$ & 353 & \\
\hline Obese & $11 \cdot 7$ & 275 & 13.9 & 164 & 9.4 & 111 & \\
\hline \multicolumn{7}{|l|}{ Pubertal maturation status (Tanner stage) } & $<0.001$ \\
\hline Stage 1 & $32 \cdot 7$ & 769 & $44 \cdot 3$ & 522 & $21 \cdot 4$ & 247 & \\
\hline Stage 2 & 41.6 & 979 & $44 \cdot 2$ & 521 & $39 \cdot 1$ & 458 & \\
\hline Stage 3 & $17 \cdot 8$ & 419 & 9.5 & 112 & $25 \cdot 9$ & 307 & \\
\hline Stage 4 & 6.6 & 155 & 1.9 & 22 & $11 \cdot 1$ & 133 & \\
\hline Stage 5 & 1.3 & 31 & 0.1 & 1 & $2 \cdot 6$ & 30 & \\
\hline \multicolumn{8}{|l|}{ Vitamin D status } \\
\hline Serum $25(\mathrm{OH}) \mathrm{D}<30 \mathrm{nmol} / \mathrm{l}$ & $5 \cdot 2$ & 122 & 3.2 & 38 & $7 \cdot 2$ & 84 & $<0.001$ \\
\hline Serum $25(\mathrm{OH}) \mathrm{D}<50 \mathrm{nmol} / \mathrm{l}$ & 52.5 & 1235 & 48.0 & 566 & $57 \cdot 0$ & 669 & $<0.001$ \\
\hline Serum $25(\mathrm{OH}) \mathrm{D}<75 \mathrm{nmol} / \mathrm{l}$ & $96 \cdot 3$ & 2266 & $95 \cdot 1$ & 1122 & 97.4 & 1144 & 0.003 \\
\hline
\end{tabular}

25(OH)D, 25-hydroxyvitamin D; PTH, parathyroid hormone.

${ }^{*} P$ values indicate sex differences and were derived from Student's $t$ test in the case of continuous variables and Pearson's $\chi^{2}$ test in the case of categorical variables.

were Caucasian and of Greek origin (83.9\%). The prevalence of obesity was higher in boys compared with girls $(P=0.001)$, whereas more girls were categorised at higher pubertal maturation stages $(P<0.001)$ and had higher prevalence rates of vitamin $\mathrm{D}$ deficiency and insufficiency compared with boys $(P<0.001$ respectively).

Table 2 presents the differences in the mean standardised serum 25(OH)D concentrations (derived by the use of the LC-MS/MS method), among different sub-groups of the study population. More specifically, mean serum 25(OH)D concentrations were found to be significantly higher in boys compared with girls $(P<0 \cdot 001)$, during autumn months compared with winter and spring months $(P<0 \cdot 001)$, in southern (Iraklio-Crete) and central (Attica) compared with western
(Aitoloakarnania) and northern (Thessaloniki) Greece $(P<0.001)$, in rural compared with large urban and semi-urban areas $(P<0 \cdot 001)$, for children whose parents had $>14$ years of education compared with those whose one or both their parents had $<14$ years of education, for children of Greek compared with those of non-Greek origin $(P=0 \cdot 011)$, for children categorised at lower compared with higher pubertal maturation stages $(P<0.001)$ and for children with lower compared with higher PTH concentrations $(P<0 \cdot 001)$.

Regarding seasonal differences, the highest prevalence rates of $25(\mathrm{OH}) \mathrm{D}<30$ and $50 \mathrm{nmol} / 1$ ( 9.1 and $73.1 \%$, respectively) were observed post-winter during spring months (from April to June), whereas the lowest ( 1.5 and $31.9 \%$, respectively) were post-summer during autumn (from October to December) 
Table 2. Serum 25-hydroxyvitamin $\mathrm{D}(25(\mathrm{OH}) \mathrm{D})$ concentrations (nmol/l) in sub-groups of the study population $(n 2353)^{*}$ (Mean values and standard deviations)

\begin{tabular}{|c|c|c|c|c|c|}
\hline \multirow[b]{2}{*}{ Sub-groups based on } & \multicolumn{2}{|c|}{$25(\mathrm{OH}) \mathrm{D}(\mathrm{nmol} / \mathrm{l})$} & \multirow[b]{2}{*}{ Sub-groups based on } & \multicolumn{2}{|c|}{ 25(OH)D (nmol/l) } \\
\hline & Mean & SD & & Mean & SD \\
\hline Sex & & & Origin & & \\
\hline Boys ( $n$ 1179) & $52 \cdot 0$ & $13 \cdot 2$ & Greek ( $n$ 1974) & $50 \cdot 4$ & $12 \cdot 9$ \\
\hline Girls ( $n$ 1174) & $48 \cdot 2$ & $12 \cdot 8$ & Non-Greek ( $n$ 379) & $48 \cdot 6$ & $14 \cdot 0$ \\
\hline$P$ & \multicolumn{2}{|c|}{$<0.001$} & $P$ & \multicolumn{2}{|c|}{0.011} \\
\hline \multicolumn{3}{|l|}{ Seasonality } & \multicolumn{3}{|l|}{ Body weight status } \\
\hline Autumn (October to December) ( $n$ 859) & $56 \cdot 4^{a, b}$ & $13 \cdot 4$ & Under-/normal-weight ( $n$ 1360) & $50 \cdot 7^{b}$ & $13 \cdot 5$ \\
\hline Winter (January to March) ( $n$ 821) & $48 \cdot 3^{\mathrm{a}, \mathrm{c}}$ & 11.7 & Overweight ( $n 718$ ) & 49.9 & $12 \cdot 6$ \\
\hline Spring (April to June) ( $n$ 673) & $44 \cdot 3^{\mathrm{b}, \mathrm{c}}$ & $11 \cdot 1$ & Obese $(n 275)$ & $47 \cdot 8^{\mathrm{b}}$ & $12 \cdot 5$ \\
\hline$P$ & \multicolumn{2}{|c|}{$<0.001$} & $P$ & \multicolumn{2}{|c|}{0.004} \\
\hline \multicolumn{3}{|l|}{ Region of residence } & \multicolumn{3}{|l|}{ Pubertal maturation status (Tanner stage) } \\
\hline Central Greece (Attica) (n 1346) & $50 \cdot 1^{a, b, c}$ & $13 \cdot 6$ & Stage $1(n 769)$ & $53 \cdot 4^{\mathrm{a}, \mathrm{b}, \mathrm{c}}$ & $13 \cdot 2$ \\
\hline Southern Greece (Iraklio-Crete) ( $n$ 398) & $56 \cdot 4^{\mathrm{a}, \mathrm{d}, \mathrm{e}}$ & 11.9 & Stage $2(n 979)$ & $49 \cdot 8^{\mathrm{a}, \mathrm{d}, \mathrm{e}}$ & 12.9 \\
\hline Western Greece (Aitoloakarnania) ( $n$ 318) & $45 \cdot 2^{b, d}$ & 11.5 & Stage 3 (n 419) & $46 \cdot 8^{\mathrm{b}, \mathrm{d}}$ & $12 \cdot 5$ \\
\hline Northern Greece (Thessaloniki) ( $n$ 291) & $47 \cdot 0^{\mathrm{c}, \mathrm{e}}$ & $10 \cdot 7$ & Stage 4 ( $n 155$ & $46 \cdot 3^{\mathrm{c}, \mathrm{e}}$ & 12.5 \\
\hline$P$ & \multicolumn{2}{|c|}{$<0.001$} & $\begin{array}{l}\text { Stage } 5 \text { (n 31) } \\
P\end{array}$ & \multicolumn{2}{|c|}{$<0.001$} \\
\hline \multicolumn{3}{|l|}{ Urbanisation degree } & \multicolumn{3}{|l|}{ Dietary vitamin $D$ intake $(\mu \mathrm{g} / \mathrm{d})$} \\
\hline Large urban regions ( $n$ 1602) & $49.5^{\mathrm{b}}$ & $13 \cdot 2$ & 1st Tertile $(<0.86)(n 786)$ & 50.5 & $13 \cdot 0$ \\
\hline Semi-urban regions ( $n 360)$ & $49 \cdot 6^{c}$ & $13 \cdot 2$ & 2nd Tertile $(0.86-1.76)(n 783)$ & $49 \cdot 4$ & $13 \cdot 0$ \\
\hline Rural regions $(n 391)$ & $53 \cdot 4^{\mathrm{b}, \mathrm{c}}$ & $12 \cdot 3$ & 3rd Tertile $(>1 \cdot 76)(n 784)$ & $50 \cdot 5$ & $13 \cdot 4$ \\
\hline$P$ & & & $P$ & & \\
\hline \multicolumn{3}{|l|}{ Parental education level } & \multicolumn{3}{|l|}{ Physical activity levels (steps/d) } \\
\hline Both parents <14 years ( $n$ 1228) & $49 \cdot 0^{\mathrm{b}}$ & $13 \cdot 0$ & 1st Tertile $(<10707)(n 786)$ & $50 \cdot 3$ & $13 \cdot 6$ \\
\hline One parent $<14$ years $(n 558)$ & $50 \cdot 2^{c}$ & $12 \cdot 4$ & 2nd Tertile (10707-14633) ( $n$ 783) & $50 \cdot 1$ & $13 \cdot 2$ \\
\hline Both parents $>14$ years $(n 567)$ & $52 \cdot 5^{\mathrm{b}, \mathrm{c}}$ & $13 \cdot 2$ & 3rd Tertile $(>14633)(n 784)$ & $50 \cdot 0$ & $12 \cdot 6$ \\
\hline$P$ & & & $P$ & & \\
\hline \multicolumn{3}{|l|}{ Race } & \multicolumn{3}{|l|}{ PTH concentrations (pg/ml) } \\
\hline Caucasian ( $n$ 2308) & $50 \cdot 2$ & $12 \cdot 9$ & 1st Tertile $(<28.9)(n$ 786) & $53 \cdot 1^{\mathrm{a}, \mathrm{b}}$ & $13 \cdot 8$ \\
\hline Non-Caucasian ( $n$ 45) & $46 \cdot 9$ & 20.5 & 2nd Tertile (28.9-38.4) ( $n$ 783) & $50 \cdot 8^{\mathrm{a}, \mathrm{c}}$ & $12 \cdot 5$ \\
\hline \multirow[t]{2}{*}{$P$} & \multirow{2}{*}{\multicolumn{2}{|c|}{0.098}} & 3rd Tertile (>38.4) ( $n$ 784) & \multirow{2}{*}{\multicolumn{2}{|c|}{$46 \cdot 0<0.001$}} \\
\hline & & & $P$ & & \\
\hline
\end{tabular}

PTH, parathyroid hormone.

a,b,c,d,e Mean values sharing the same superscript letter are significantly different between groups as derived from pairwise (post hoc) comparisons using the Bonferroni rule for Type 1 error correction.

* All $P$ values were derived from Student's $t$ test or ANOVA.

(Fig. 1(a) and (b)). The increasing trend observed in the prevalence of low vitamin D status, from autumn to winter and spring months, was statistically significant $(P<0.05)$.

According to the data presented in Fig. 2, the prevalence rates of vitamin D deficiency and insufficiency were higher among children from urban and semi-urban regions examined during autumn ( $2 v .0 \%$ and $33.8 v .25 \cdot 0 \%$, respectively; $P \leq 0.05)$ and spring (9.6 v. $0 \%$ and 74.6 v. $47.2 \%$, respectively; $P<0.05$ ), compared with children from rural regions examined during the same time periods. No statistically significant urban/rural differences were observed during winter months. It should be noted that as the current study was school-based, blood samples were not collected from mid-June to mid-September due to the summer break. As such, no data on vitamin D status are available for the summer period.

The OR and 95\% CI for vitamin D deficiency and insufficiency in schoolchildren, stratified by sex, degree of urbanisation and seasonality (and after adjusting for several potential confounding factors) are shown in Table 3. Based on this data, girls were found to be 1.79 (95\% CI 1.15, 2.80) and 1.24 (95\% CI 1.02, 1.52) times more likely to be vitamin D insufficient and deficient, respectively, compared with boys. In addition, children measured during winter and spring months had the highest odds for vitamin D insufficiency (4.53; $95 \%$ CI 1.93, $10 \cdot 6$ and $7 \cdot 47 ; 95 \%$ CI 3.23, $17 \cdot 3$ respectively) and deficiency (2.38; $95 \%$ CI $1.81,3 \cdot 13$ and 5.14; $95 \%$ CI 3.84, 6.89 respectively), compared with children measured during autumn months. Lastly, large urban and semi-urban region of residence of children was associated with increased likelihoods for vitamin D insufficiency (2.88; $95 \%$ CI 1.28, 6.51) and deficiency (1.49; $95 \%$ CI 1.16, 1.93), compared with children living in rural regions.

\section{Discussion}

The available literature on vitamin D status in populations of children and adolescents in Europe is characterised by a high degree of variability among countries and by relatively high prevalence rates of vitamin D deficiency and insufficiency, even in southern European countries ${ }^{(6,11,29)}$. This high degree of variability observed among European countries is partly explained by the different biochemical assays and thresholds used to assess vitamin D status ${ }^{(29-31)}$. This is the largest analysis of vitamin D status among primary schoolchildren in Greece. The overall prevalence of $52.5 \%$ for vitamin D insufficiency reported for children in Greece by the present 


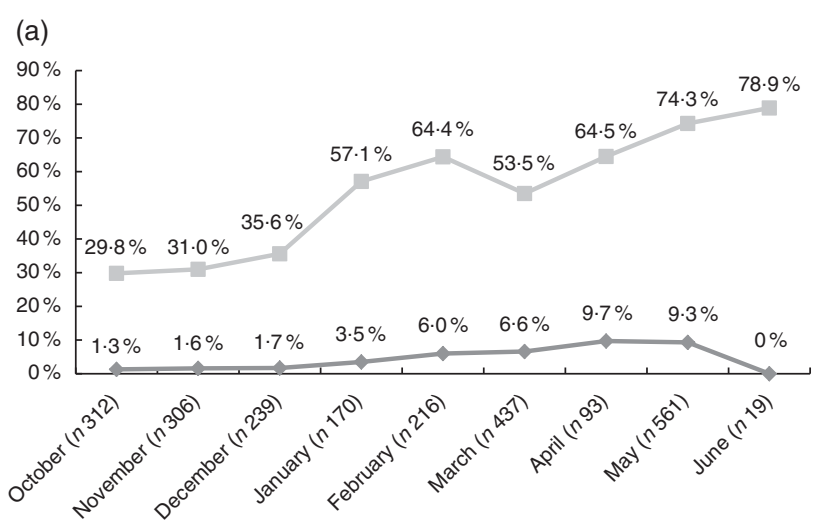

(b)

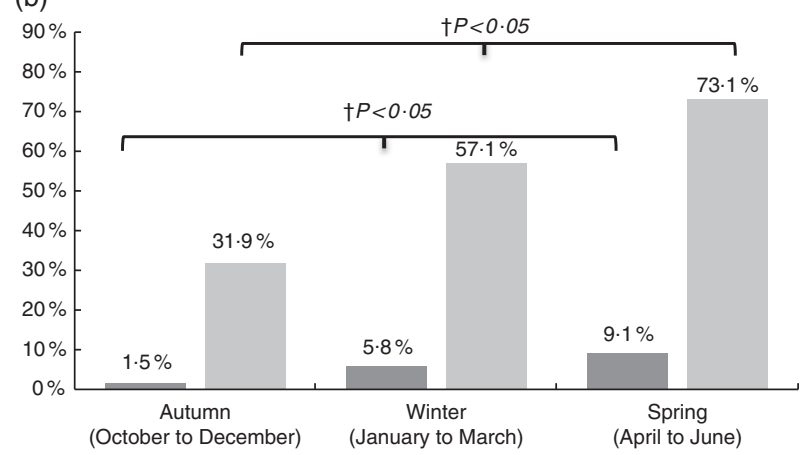

Fig. 1. Prevalence of vitamin $D$ deficiency (25-hydroxyvitamin $D(25(\mathrm{OH}) \mathrm{D})$ $<30 \mathrm{nmol} / \mathrm{l})$ and insufficiency $(25(\mathrm{OH}) \mathrm{D}<50 \mathrm{nmol} / \mathrm{l})$ by month (a) and by season (b). a: $\longrightarrow, 25(\mathrm{OH}) \mathrm{D}<30 \mathrm{nmol} / \mathrm{l} ;-\square, 25(\mathrm{OH}) \mathrm{D}<50 \mathrm{nmol} / \mathrm{l}$; b: $\square, 25(\mathrm{OH}) \mathrm{D}<30 \mathrm{nmol} / / ; \square, 25(\mathrm{OH}) \mathrm{D}<50 \mathrm{nmol} / \mathrm{l}$. $\dagger P$ values indicate seasonal differences and have derived from the Pearson's $x^{2}$ test, using the two-sample test for proportions for post hoc multiple comparisons.

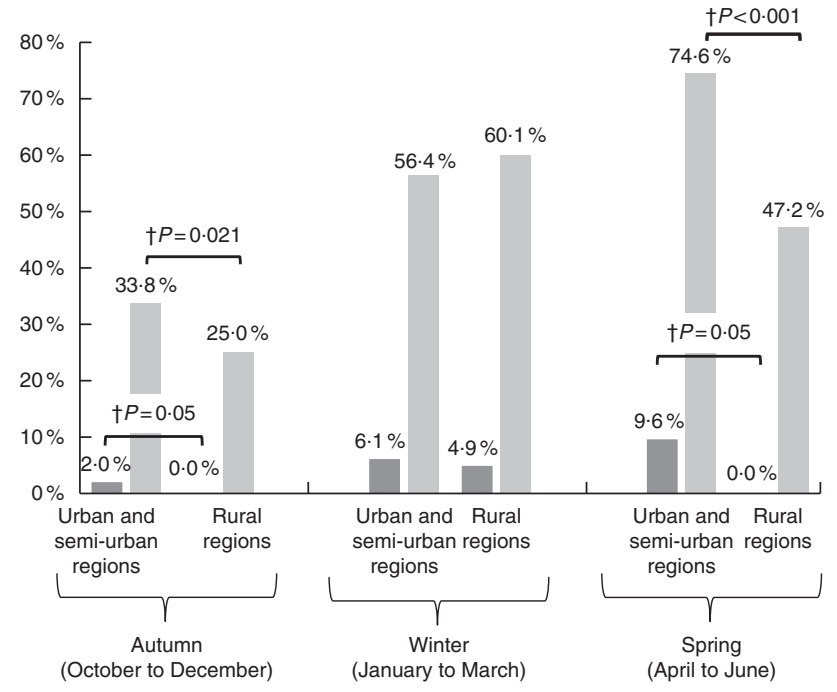

Fig. 2. Prevalence of vitamin $D$ deficiency (25-hydroxyvitamin $D(25(\mathrm{OH}) \mathrm{D})$ $<30 \mathrm{nmol} / \mathrm{l}, \square)$ and insufficiency $(25(\mathrm{OH}) \mathrm{D}<50 \mathrm{nmol} / \mathrm{l}, \square)$ by urbanisation degree in the three seasonally-stratified sub-groups. $\dagger P$ values indicate regional differences and have derived from the Pearson's $x^{2}$ test, using the twosample test for proportions for post hoc multiple comparisons.

study is intermediate to the relevant prevalence rates of 45.9 and $82.3 \%$ recorded for children and adolescents living in two other Mediterranean countries, namely Italy and Turkey respectively ${ }^{(13,14)}$. However, this prevalence remains higher or very similar to the relevant proportions of vitamin D insufficiency reported by other studies on children and adolescents in central and northern European countries ${ }^{(15-17,32)}$

Although Greece is a Mediterranean country with adequate sunshine and UVB availability in most months over the year ${ }^{(33)}$ and despite the fact that the major source of vitamin $D$ is its endogenous synthesis via skin exposure to solar UVB radiation, several environmental, personal and lifestyle factors can substantially affect vitamin D status. In this regard, the current study reported sex, regional and seasonal differences in the prevalence of vitamin D status among children. More specifically, our data showed that mean serum 25(OH)D concentrations were significantly higher in boys compared with girls (i.e. by a mean difference of $3.8 \mathrm{nmol} / \mathrm{l}$ ) and furthermore the prevalence rates of both vitamin D deficiency and insufficiency were significantly higher in girls compared with boys. The lower vitamin D status observed in the present study for girls in comparison with boys is also confirmed by several other studies conducted with children and/or adolescents in other European countries, but also in the USA, Australia and New Zealand $^{(17,34-39)}$. Although dietary vitamin D intake was not exceeding the recommended Estimated Average Requirement (EAR) value in both sexes, the present study showed a higher mean dietary intake of vitamin D in boys compared with girls, which could partly explain the relevant sex differences observed in vitamin D status. In the same context, as indicated by the Third National Health and Nutrition Examination Survey (NHANES III) $^{(37)}$, the lower dietary intake of vitamin D recorded for girls compared with boys in the USA could be one factor supporting the sex differences in vitamin D status. In addition to diet, the lower levels of physical activity usually reported for girls compared with boys in childhood and adolescence may also explain the sex differences observed in the present and other studies, with regards to the prevalence of vitamin D deficiency and insufficiency. Physical activity executed outdoors is usually a proxy measure of sunlight exposure and as such of cutaneous synthesis of vitamin D. However, in the present study female sex remained positively associated with vitamin D deficiency and insufficiency, even after adjusting for children's dietary intake of vitamin D and physical activity levels but also for other potential confounding factors, such as BMI, pubertal maturation, socio-demographic characteristics and serum PTH concentrations ${ }^{(39-41)}$, thus implying the possible role of additional factors that might mediate this association.

Regarding seasonal differences, as expected the highest and lowest prevalence rates of vitamin D deficiency and insufficiency were observed during typical post-winter (April to June) and postsummer (October to December) months, respectively. This is indicative of a clear and most likely independent effect of season on children's vitamin D status, considering the increased likelihood of vitamin D deficiency and insufficiency observed in the present study during winter and spring months, even after adjusting for several potential confounding factors. In this regard mean serum $25(\mathrm{OH}) \mathrm{D}$ concentrations usually follow a sinusoidal pattern throughout the year, reaching their zenith at the post-summer period/beginning of autumn and their nadir at the post-winter period/beginning of spring in populations of the 
Table 3. Multivariate logistic regression analyses examining the associations of vitamin $D$ deficiency and insufficiency (i.e. 25-hydroxyvitamin $D$ levels $<30$ and $50 \mathrm{nmol} /$, respectively) with sex, seasonality and urbanisation degree*

(Odds ratios and $95 \%$ confidence intervals)

\begin{tabular}{|c|c|c|c|c|c|c|}
\hline & \multicolumn{3}{|c|}{$\begin{array}{l}\text { Vitamin D deficiency } \\
(<30 \mathrm{nmol} / \mathrm{l})\end{array}$} & \multicolumn{3}{|c|}{$\begin{array}{l}\text { Vitamin D insufficiency } \\
(<50 \mathrm{nmol} / \mathrm{l})\end{array}$} \\
\hline & OR & $95 \% \mathrm{Cl}$ & $P$ & OR & $95 \% \mathrm{Cl}$ & $P$ \\
\hline \multicolumn{7}{|l|}{ Sex } \\
\hline Boys & 1.00 & & & 1.00 & & \\
\hline Girls & 1.79 & $1 \cdot 15,2 \cdot 80$ & 0.011 & 1.24 & $1.02,1.52$ & 0.031 \\
\hline \multicolumn{7}{|l|}{ Seasonality } \\
\hline Autumn & 1.00 & & & 1.00 & & \\
\hline Winter & 4.53 & $1.93,10 \cdot 6$ & 0.001 & $2 \cdot 38$ & $1.81,3 \cdot 13$ & $<0.001$ \\
\hline Spring & 7.47 & $3 \cdot 23,17 \cdot 3$ & $<0.001$ & $5 \cdot 14$ & $3.84,6.89$ & $<0.001$ \\
\hline \multicolumn{7}{|l|}{ Urbanisation degree } \\
\hline Rural & 1.00 & & & 1.00 & & \\
\hline Urban/semi-urban & 2.88 & $1.28,6.51$ & 0.001 & 1.49 & $1.16,1.93$ & $<0.001$ \\
\hline
\end{tabular}

northern hemisphere ${ }^{(42)}$. These findings are consistent with several other relevant studies available in the literature, conducted with children from other Mediterranean countries ${ }^{(13,14,34,35)}$.

One recent study conducted with adults living in the island of Crete in southern Greece ${ }^{(43)}$, showed much higher serum 25(OH)D during spring months, compared with the present study. Possible explanations for this inconsistency could stem from the differences between the two studies, in terms of the examined age groups and latitude. In this regard, the study in Crete examined mainly adults, $34 \%$ of whom were inpatients in local hospitals, whereas the southern latitude of the island Crete facilitates skin exposures to sunlight for more months throughout the year compared with the northern latitudes of mainland Greece. On the contrary the present study was conducted with children, who spend a great proportion of their daily time indoors as they have to attend school until mid-June (thus probably explaining the lowest serum $25(\mathrm{OH}) \mathrm{D}$ levels observed in the present study from April to mid-June), whereas the study population was recruited from four different regions, covering the southern, central, western and northern parts of Greece. Last but not least, as the present study was school-based, measurements were not conducted during typical summer months in Greece (i.e. mid-June to mid-September) when a gradual increase in mean $25(\mathrm{OH}) \mathrm{D}$ levels should be expected in agreement with the data reported by the study performed in Crete.

As far as the role of urbanisation degree on vitamin D status is concerned, the present study showed higher prevalence rates of vitamin D deficiency and insufficiency for children living in urban/semi-urban regions compared with their counterparts from rural regions, especially during autumn and spring months. These differences in vitamin D status among children living in areas of different urbanisation degree could mainly be attributed to the particularities of the physical environment in urban compared with rural regions of Greece, as determinant of behaviours also affecting vitamin D status. More specifically, the physical environment in rural regions can be more supportive of increased outdoor physical activity levels and consequently greater sun exposure compared with urban and semi-urban regions $^{(44)}$. Typical examples of how physical environment can facilitate optimal $25(\mathrm{OH}) \mathrm{D}$ levels in rural compared with urban areas are the higher availability of and accessibility to open-space areas, less traffic and more neighbourhood safety supporting active transportation, shorter buildings and less or no air pollution, which can inhibit endogenous/cutaneous synthesis of vitamin D by blocking direct sun exposure. These regional differences in vitamin $\mathrm{D}$ status were mainly seen at post-summer and post-winter time periods, when prevailing weather conditions in Greece are more supportive for outdoor playing and activities. On the other hand, no regional differences on vitamin D status were observed during typical winter months (January to March), as at this time period children from both urban and rural regions usually spent more time indoors, due to the colder weather conditions and shorter daytime, whereas at the same time sun exposure during this period is not efficient enough in stimulating endogenous vitamin D synthesis ${ }^{(45)}$.

The findings of the current study should be interpreted in light of its strengths and limitations. Regarding strengths, the current study was a large-scale epidemiological study conducted in a representative sample of children from four prefectures within the wider region of Greece. Furthermore, standardisation of serum $25(\mathrm{OH}) \mathrm{D}$ concentrations based on the VDSP protocols is a novel approach that sets the basis for more consistent and valid comparisons with current and all future studies that will use the 'gold standard' LC-MS/MS assay to assess vitamin status. Regarding limitations, the low dietary vitamin $\mathrm{D}$ intake reported by the present study could reflect the weaknesses in the method used to record children's dietary intake (i.e. $24 \mathrm{~h}$ recall) and/or in the dietary intake analysis software and the food composition tables in terms of the actual food sources of vitamin D.

In conclusion, the prevalence of low vitamin $\mathrm{D}$ status reported in the present study for schoolchildren in Greece is considerably high and is comparable to, or in many cases exceeds, the relevant prevalence reported for children and adolescents in other European countries. Girls, children living in 
urban regions and post-winter months were found to significantly increase the likelihood of vitamin D deficiency and insufficiency thus highlighting specific sub-groups living in certain physical environments and under specific conditions that might benefit the most from population-based strategies aiming to tackle the actual problem of low vitamin D status. Among such strategies, dietary intake of vitamin D from fortified foods could be considered as the one with probably the widest population reach for maintaining sufficient levels of vitamin D in children. This is further supported by the fact that vitamin D food fortification in Greece is optional and as a consequence dietary intake of vitamin $\mathrm{D}$ has been repeatedly reported to be inadequate, not only for children but for adults too ${ }^{(46)}$.

\section{Acknowledgements}

The authors would like to thank all research members involved in the data collection of the Healthy Growth Study.

The biochemical analyses for determining serum 25-hydroxyvitamin D concentrations were partly funded by Kellogg Company Europe and partly by Roche Diagnostics SA. The standardisation of serum 25-hydroxyvitamin D concentrations was supported in part by funding received from the European Commission under its Seventh Framework Programme (FP7) ODIN (grant agreement no. 613977). Roche Diagnostics SA had no role in the design, analysis or writing of this article.

Y. M. and G. M. contributed in designing and monitoring the study, in collecting and managing the data and in leading the statistical analyses with the contribution and input from T. H. and A.-S. B.; G. L. J. H. and K. G. D. conducted the LC-MS/MS re-analysis of sera for VDSP standardisation. K. D. C. and M. E. K. performed the uniform sampling as per VDSP procedures to identify serum samples for re-analysis and developed the master regression equations. All authors contributed to the writing, critical review and approval of the final version of the manuscript for publication.

M. E. K. and K. D. C. are joint coordinators of the European Commission-funded ODIN integrated project (contract 613977). T. H. is and A.-S. B. was an employee at Kellogg Company. Any opinions, findings, conclusions or recommendations expressed in the current study are those of the authors and do not necessarily reflect the views of Kellogg Company.

\section{Supplementary material}

For supplementary material/s referred to in this article, please visit https://doi.org/10.1017/S0007114517002422

\section{References}

1. Braegger C, Campoy C, Colomb V, et al. (2013) Vitamin D in the healthy paediatric population: a position paper by the ESPGHAN Committee on Nutrition. J Pediatr Gastroenterol Nutr 56, 692-701.

2. Misra M, Pacaud D, Petryk A, et al. (2008) Vitamin D deficiency in children and its management: review of current knowledge and recommendations. Pediatrics 122, 398-417.
3. Taylor CL, Thomas PR, Aloia JF, et al. (2015) Questions about vitamin $\mathrm{D}$ for primary care practice: input from an NIH conference. Am J Med 128, 1167-1170.

4. Cashman KD \& Kiely M (2011) Towards prevention of vitamin $\mathrm{D}$ deficiency and beyond: knowledge gaps and research needs in vitamin D nutrition and public health. BrJ Nutr 106, $1617-1627$

5. Theodoratou E, Tzoulaki I, Zgaga L, et al. (2014) Vitamin D and multiple health outcomes: umbrella review of systematic reviews and meta-analyses of observational studies and randomised trials. BMJ 348, g2035.

6. van Schoor NM \& Lips P (2011) Worldwide vitamin D status. Best Pract Res Clin Endocrinol Metab 25, 671-680.

7. Hagenau T, Vest R, Gissel TN, et al. (2009) Global vitamin D levels in relation to age, gender, skin pigmentation and latitude: an ecologic meta-regression analysis. Osteoporos Int 20, 133-140.

8. Lips P (2007) Vitamin D status and nutrition in Europe and Asia. J Steroid Biochem Mol Biol 103, 620-625.

9. Institute of Medicine Food and Nutrition Board (2011) Dietary reference intakes for calcium and vitamin D. Washington, DC: National Academy Press.

10. Holick MF, Binkley NC, Bischoff-Ferrari HA, et al. (2011) Evaluation, treatment, and prevention of vitamin D deficiency: an Endocrine Society clinical practice guideline. J Clin Endocrinol Metab 96, 1911-1930.

11. Hilger J, Friedel A, Herr R, et al. (2014) A systematic review of vitamin D status in populations worldwide. Br J Nutr 111, $23-45$.

12. Gonzalez-Gross M, Valtuena J, Breidenassel C, et al. (2012) Vitamin D status among adolescents in Europe: the Healthy Lifestyle in Europe by Nutrition in Adolescence study. Br J Nutr 107, 755-764.

13. Karaguzel G, Dilber B, Can G, et al. (2014) Seasonal vitamin $D$ status of healthy schoolchildren and predictors of low vitamin D status. J Pediatr Gastroenterol Nutr 58, 654-660.

14. Vierucci F, Del Pistoia M, Fanos M, et al. (2013) Vitamin D status and predictors of hypovitaminosis D in Italian children and adolescents: a cross-sectional study. Eur $J$ Pediatr 172, 1607-1617.

15. Voortman T, van den Hooven EH, Heijboer AC, et al. (2015) Vitamin D deficiency in school-age children is associated with sociodemographic and lifestyle factors. J Nutr 145 , 791-798.

16. Absoud M, Cummins C, Lim MJ, et al. (2011) Prevalence and predictors of vitamin D insufficiency in children: a Great Britain population based study. PLOS ONE 6, e22179.

17. Hill TR, Cotter AA, Mitchell S, et al. (2008) Vitamin D status and its determinants in adolescents from the Northern Ireland Young Hearts 2000 cohort. Br J Nutr 99, 1061-1067.

18. Aypak C, Turedi O \& Yuce A (2014) The association of vitamin D status with cardiometabolic risk factors, obesity and puberty in children. Eur J Pediatr 173, 367-373.

19. Akman AO, Tumer L, Hasanoglu A, et al. (2011) Frequency of vitamin D insufficiency in healthy children between 1 and 16 years of age in Turkey. Pediatr Int 53, 968-973.

20. Sempos CT, Vesper HW, Phinney KW, et al. (2012) Vitamin D status as an international issue: national surveys and the problem of standardization. Scand J Clin Lab Invest Suppl $\mathbf{2 4 3}, 32-40$.

21. Carter GD, Carter R, Jones J, et al. (2004) How accurate are assays for 25-hydroxyvitamin D? Data from the international vitamin D external quality assessment scheme. Clin Chem 50, 2195-2197.

22. Binkley N \& Sempos CT (2014) Standardizing vitamin D assays: the way forward. J Bone Miner Res 29, 1709-1714. 
23. Moschonis G, Tanagra S, Vandorou A, et al. (2010) Social, economic and demographic correlates of overweight and obesity in primary-school children: preliminary data from the Healthy Growth Study. Public Health Nutr 13, 1693-1700.

24. University of Crete (1991) Food composition tables nutrition. med.uoc.gr/GreekTables (accessed March 2017).

25. Trichopoulou A (2004) Composition Tables of Foods and Greek Dishes. Athens: Department of Hygiene and Epidemiology, School of Medicine.

26. Tanner JM (1955) Growth at Adolescence. Oxford: Blackwell Scientific.

27. Cashman KD, Kiely M, Kinsella M, et al. (2013) Evaluation of Vitamin D Standardization Program protocols for standardizing serum 25-hydroxyvitamin D data: a case study of the program's potential for national nutrition and health surveys. Am J Clin Nutr 97, 1235-1242.

28. Cashman KD, Dowling KG, Skrabakova Z, et al. (2015) Standardizing serum 25-hydroxyvitamin $D$ data from four Nordic population samples using the Vitamin D Standardization Program protocols: Shedding new light on vitamin D status in Nordic individuals. Scand J Clin Lab Invest $\mathbf{7 5}$, 549-561.

29. Cashman KD, Dowling KG, Skrabakova Z, et al. (2016) Vitamin D deficiency in Europe: pandemic? Am J Clin Nutr 103, 1033-1044.

30. Binkley N, Krueger D, Cowgill CS, et al. (2004) Assay variation confounds the diagnosis of hypovitaminosis D: a call for standardization. J Clin Endocrinol Metab 89, 3152-3157.

31. Wallace AM, Gibson S, de la Hunty A, et al. (2010) Measurement of 25-hydroxyvitamin $\mathrm{D}$ in the clinical laboratory: current procedures, performance characteristics and limitations. Steroids 75, 477-488.

32. Sioen I, Mouratidou T, Kaufman JM, et al. (2012) Determinants of vitamin D status in young children: results from the Belgian arm of the IDEFICS (Identification and Prevention of Dietaryand Lifestyle-Induced Health Effects in Children and Infants) Study. Public Health Nutr 15, 1093-1099.

33. O'Neill CM, Kazantzidis A, Ryan MJ, et al. (2016) Seasonal changes in vitamin D-effective UVB availability in Europe and associations with population serum 25-hydroxyvitamin D. Nutrients 8, E533-E547.

34. Kolokotroni O, Papadopoulou A, Yiallouros PK, et al. (2015) Association of vitamin D with adiposity measures and other determinants in a cross-sectional study of Cypriot adolescents. Public Health Nutr 18, 112-121.
35. Tolppanen AM, Fraser A, Fraser WD, et al. (2012) Risk factors for variation in 25-hydroxyvitamin $\mathrm{D}(3)$ and $\mathrm{D}(2)$ concentrations and vitamin D deficiency in children. J Clin Endocrinol Metab 97, 1202-1210.

36. Dong Y, Pollock N, Stallmann-Jorgensen IS, et al. (2010) Low 25-hydroxyvitamin D levels in adolescents: race, season, adiposity, physical activity, and fitness. Pediatrics $\mathbf{1 2 5}$, 1104-1111.

37. Kumar J, Muntner P, Kaskel FJ, et al. (2009) Prevalence and associations of 25-hydroxyvitamin D deficiency in US children: NHANES 2001-2004. Pediatrics 124, e362-e370.

38. Black LJ, Burrows SA, Jacoby P, et al. (2014) Vitamin D status and predictors of serum 25-hydroxyvitamin D concentrations in Western Australian adolescents. . BrJ Nutr 112, 1154-1162.

39. Rockell JE, Green TJ, Skeaff CM, et al. (2005) Season and ethnicity are determinants of serum 25-hydroxyvitamin D concentrations in New Zealand children aged 5-14 y. J Nutr 135, 2602-2608.

40. Touvier M, Deschasaux M, Montourcy M, et al. (2015) Determinants of vitamin D status in Caucasian adults: influence of sun exposure, dietary intake, sociodemographic, lifestyle, anthropometric, and genetic factors. J Invest Dermatol 135, 378-388.

41. Gutierrez Medina S, Gavela-Perez T, Dominguez-Garrido MN, et al. (2015) The influence of puberty on vitamin D status in obese children and the possible relation between vitamin $\mathrm{D}$ deficiency and insulin resistance. J Pediatr Endocrinol Metab 28, 105-110.

42. Shoben AB, Kestenbaum B, Levin G, et al. (2011) Seasonal variation in 25-hydroxyvitamin $\mathrm{D}$ concentrations in the cardiovascular health study. Am J Epidemiol 174, 1363-1372.

43. Katrinaki M, Kampa M, Margioris A, et al. (2016) Vitamin D levels in a large Mediterranean cohort: reconsidering normal cut-off values. Hormones (Athens) 15, 205-223.

44. Lucas RM, Ponsonby AL, Dear K, et al. (2013) Vitamin D status: multifactorial contribution of environment, genes and other factors in healthy Australian adults across a latitude gradient. J Steroid Biochem Mol Biol 136, 300-308.

45. Holick MF (2011) Vitamin D: a d-lightful solution for health. J Investig Med 59, 872-880.

46. Manios Y, Moschonis G, Mavrogianni C, et al. (2014) Micronutrient intakes among children and adults in Greece: the role of age, sex and socio-economic status. Nutrients 6, 4073-4092. 